> La chromatine, formée de l'enroulement d'ADN autour d'un cœur d'histones, s'organise suivant différents niveaux de compaction: de la particule cœur de nucléosome (NCP pour nucleosome core particle) à l'organisation en chromosomes dans le noyau. La structure du premier niveau d'organisation de la chromatine, la NCP, a été déterminée sans ambiguïté par cristallographie. L'organisation de la chromatine est dynamique et régulée par de nombreux facteurs pour que l'ADN soit accessible et la chromatine fonctionnelle. Notamment, des modifications post-traductionnelles des histones altèrent les charges de leurs extrémités amino- et carboxy-terminales et sont ainsi susceptibles de réguler le degré de compaction de la chromatine. Des facteurs de remodelage permettent également le glissement relatif de l'ADN autour des histones et augmentent son accessibilité. <

\section{Structure et dynamique de la particule cour de nucléosome}

Aurélie Bertin, Stéphanie Mangenot

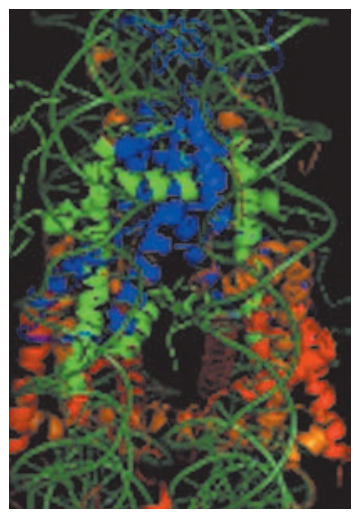

A. Bertin : University of California Berkeley, Molecular and Cellular Biology Department, Berkeley CA 94720-3220, États-Unis.

S. Mangenot :

Université Paris-Sud, 15, rue Georges Clémenceau, 91405 Orsay Cedex, France. Institut Curie, UMRI68-CNRS, 26, rue d'Ulm, 75248 Paris Cedex 05, France stephanie.mangenot@curie.fr
Chaque cellule eucaryote, cellule qui par définition possède un noyau, doit stocker des quantités colossales d'information génétique portées par de I'ADN (acide désoxyribo-nucléique) nucléaire. Le génome humain comporte trois milliards de paires de base d'ADN qui mises bout à bout, s'assemblent sur environ un mètre de long. Le mètre d'ADN humain, réparti en 46 chromosomes, est confiné dans un noyau de dimensions micrométriques (entre 1 et 10 micromètres environ). Dans un tampon physiologique (150 mM en sels monovalents), chaque molécule d'ADN formerait une pelote de $100 \mu \mathrm{m}$ de diamètre dont les dimensions seraient très supérieures à celles du noyau. Pour assurer un tel degré de compaction, l'ADN n'est pas nu mais s'assemble en une structure complexe dénommée chromatine, constituée d'ADN, de protéines basiques appelées histones et de protéines non histones, généralement plus grosses que les histones, et présentes en quantité plus faible. Le rôle de ces protéines non histones ne sera pas discuté en détails dans cet article, mais l'on peut citer à titre d'exemple les protéines 1 de l'hétérochromatine (HPl) [1], ou les protéines SMC (structural maintenance of chromosomes) [2]. Malgré un degré de compaction extrêmement important, I'ADN doit être accessible à toute la machinerie transcriptionnelle qui contrôle sa réplication, sa transcription et sa réparation. C'est pour assurer cette accessibilité que la chromatine doit subir des transitions structurales dynamiques.

\section{La particule cœur de nucléosome}

Le premier niveau d'organisation de la chromatine L'ADN, support de l'information génétique, est formé par l'association antiparallèle de deux brins polymériques de nucléotides (Figure 1). Chaque nucléotide est constitué par un sucre, un phosphate et une base (purines: adénine $(A)$ et guanine $(G)$, pyrimidines: thymine $(T)$ et cytosine $(C)$ ). Ces deux brins polymériques s'agencent en double hélice droite via des liaisons hydrogènes entre bases complémentaires $A-T$ et $C-G$. L'ADN double brin sous la forme $B$ canonique comporte 10,5 paires de bases par tour et a un diamètre de $20 \AA$. L'espacement entre deux paires de bases consécutives est de 3,4 $\mathrm{A}$. La présence de groupements phosphates en fait un polymère chargé, un polyélectrolyte dont la densité linéique de charges est de une charge négative par 1,7 $\AA$. Ce polymère est semi-flexible. Dans des conditions salines «physiologiques», la longueur de persistance de l'ADN (longueur suivant laquelle l'ADN peut être considéré comme rigide) est de l'ordre de $50 \mathrm{~nm}$ (150 paires de bases). 
Les histones sont globalement chargées positivement par la présence de nombreux acides aminés arginine et lysine. On distingue deux catégories distinctes d'histones. Les histones de cœur $\mathrm{H} 2 \mathrm{~A}, \mathrm{H} 2 \mathrm{~B}, \mathrm{H} 3$ et $\mathrm{H} 4$ sont associées à l'ADN pour former les particules cœur de nucléosome. Le repliement de l'histone $\mathrm{H} 3$ au sein de la particule cœur de nucléosome est présenté sur la Figure 2. Les histones de liaison ( $\mathrm{Hl}$ ou H5) interviennent dans des niveaux d'organisation supérieurs de la chromatine. Les histones de cœur ont une masse moléculaire d'environ 10 à $15 \mathrm{kDa}$, plus faible que celle des histones de liaison (21 kDa).

La séquence en acides aminés des histones $\mathrm{H} 2 \mathrm{~A}, \mathrm{H} 2 \mathrm{~B}, \mathrm{H} 3$ et $\mathrm{H} 4$ a été remarquablement bien conservée au cours de l'évolution. La séquence d'histone $\mathrm{H} 4$ de thymus de veau ne diffère par exemple que de deux acides aminés de celle du petit pois [3]. La région la plus conservée de ces histones est leur domaine central structuré composé du «motif histone fold » qui comprend trois hélices séparées par deux boucles (Figure 2). En revanche, les extrémités amino- et carboxy-terminales de ces histones, aussi appelées «queues des histones» sont plus variables et non structurées. Ces extrémités sont le site des modifications post-traductionnelles que nous décrirons par la suite. Dans des conditions ioniques physiologiques, les histones $\mathrm{H} 3$ et $\mathrm{H} 4$ s'associent en tétramères $(\mathrm{H} 3-\mathrm{H} 4) 2$ alors que les histones $\mathrm{H} 2 \mathrm{~A}$ et $\mathrm{H} 2 \mathrm{~B}$ forment des dimères. À forte concentration en sels monovalents (>1,2 M), les histones s'associent en octamères dans une organisation tripartite : un tétramère $(\mathrm{H} 3-\mathrm{H} 4)_{2}$ et deux dimères $\mathrm{H} 2 \mathrm{~A}-\mathrm{H} 2 \mathrm{~B}$.

Avant 1970, les histones étaient considérées comme liées uniformément à I'ADN sans organisation particulière dans un modèle de surenroulement [4]. En 1973, Hewish et Burgoyne montrent que l'ADN extrait de noyaux de foies de rats traités à la nucléase est digéré en

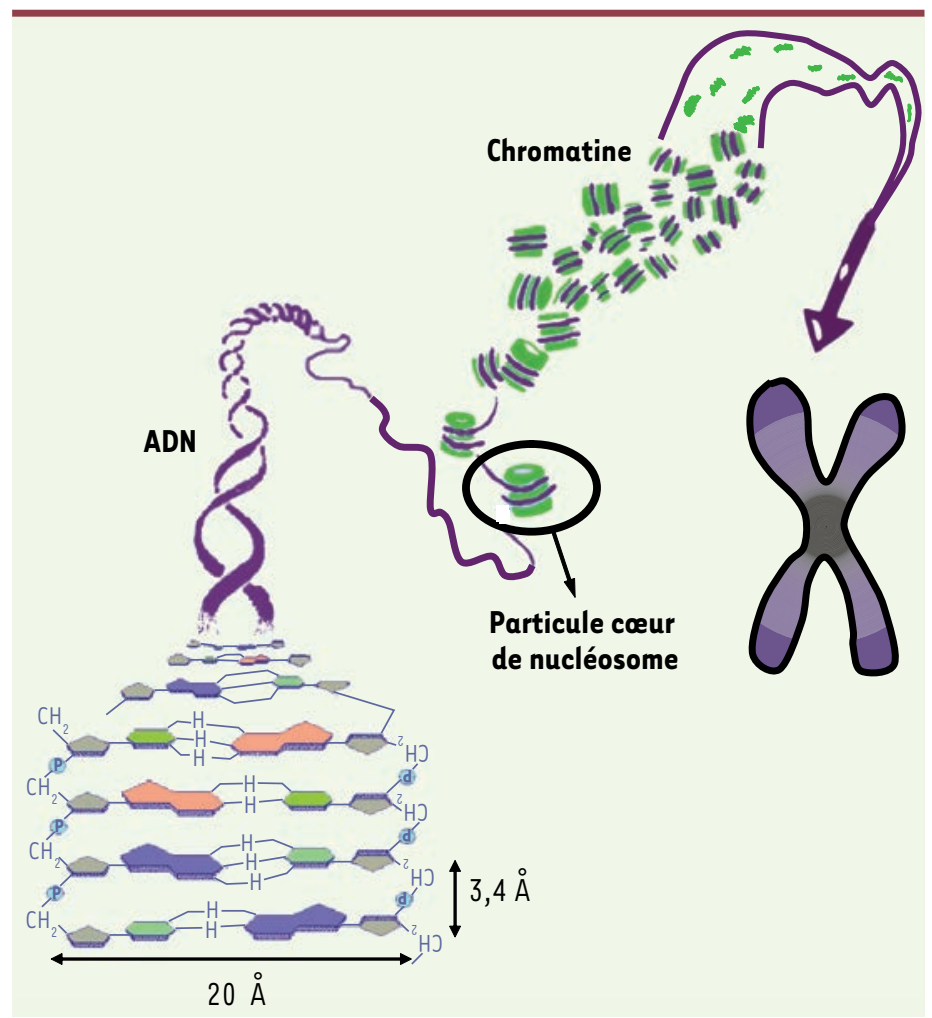

unités de taille égale [5]. En 1974, Kornberg démontre la structure périodique d'un complexe nucléoprotéique [6]. En janvier 1974, Olins et Olins publient pour la première fois des images de microscopie électronique où une structure en «collier de perles » représentative de la chromatine est mise en évidence [7]. Chaque perle constitue une particule cœur de nucléosome (NCP, nucleosome core particle). Ce complexe nucléoprotéique a une masse moléculaire d'environ 205 kDa, ADN et histones contribuant chacun pour moitié à la masse moléculaire totale. Une première structure cristallographique de nucléosomes a été obtenue à $7 \AA$ de résolution à partir de complexes natifs dont la longueur d'ADN associée aux histones s'élevait à $146 \pm 2$ pb [8].

La structure cristallographique des NCP a été résolue il y a presque 10 ans à $2,8 \AA$ de résolution [9]. L'obtention de cette structure a nécessité l'utilisation d'ADN et d'histones recombinants afin d'obtenir des particules parfaitement identiques et par conséquent facilitant l'obtention de cristaux diffractants à plus haute résolution. La Figure 3A illustre cette structure. Les NCP sont constituées d'un octamère d'histones, formés par l'assemblage du tétramère $(\mathrm{H} 3-\mathrm{H} 4)_{2}$ flanqué par deux dimères $\mathrm{H} 2 \mathrm{~A}-\mathrm{H} 2 \mathrm{~B}$, autour duquel s'enroule sur un tour trois quart, en une hélice gauche, une longueur d'ADN de 146 paires de bases. Ces particules possèdent un pseudo-axe de symétrie binaire ou axe dyade. Les NCP ainsi cristallisées ne sont pas complètement symétriques. Une moitié comporte 73 pb alors que la moitié complémentaire n'en comprend que 72, l'axe de symétrie traversant une base. En moyenne, l'ADN des NCP cristallisées comporte 10,2 pb par tour, ce qui est inférieur de 0,3 pb à un ADN B canonique. L'ADN subit donc un surenroulement global qui est particulièrement remarquable au sein de la moitié qui ne comptabilise que 72 pb où sont dénombrées 9,4 pb par tour d'ADN. La longueur d'ADN enroulé autour de l'octamère d'histones correspond exactement à la longueur de persistance de l'ADN. Des interactions privilégiées doivent donc être mises en œuvre entre octamère d'histones et ADN afin de parvenir à courber la molécule d'ADN et à maintenir

Figure 1. Appariement des paires de bases complémentaires pour former la structure en hélice droite de l'ADN. Au sein des cellules eucaryotes, 146 paires de bases d'ADN s'enroulent en une hélice gauche sur un tour trois quart autour d'un cœur de protéines, appelées histones, pour former la particule cœur de nucléosome ou NCP. Ces particules sont séparées entre elles par des portions d'ADN nu appelé ADN linker pour former la structure en collier de perles de la chromatine. Cette structure est ensuite compactée en des niveaux d'organisation supérieurs toujours en discussion. 


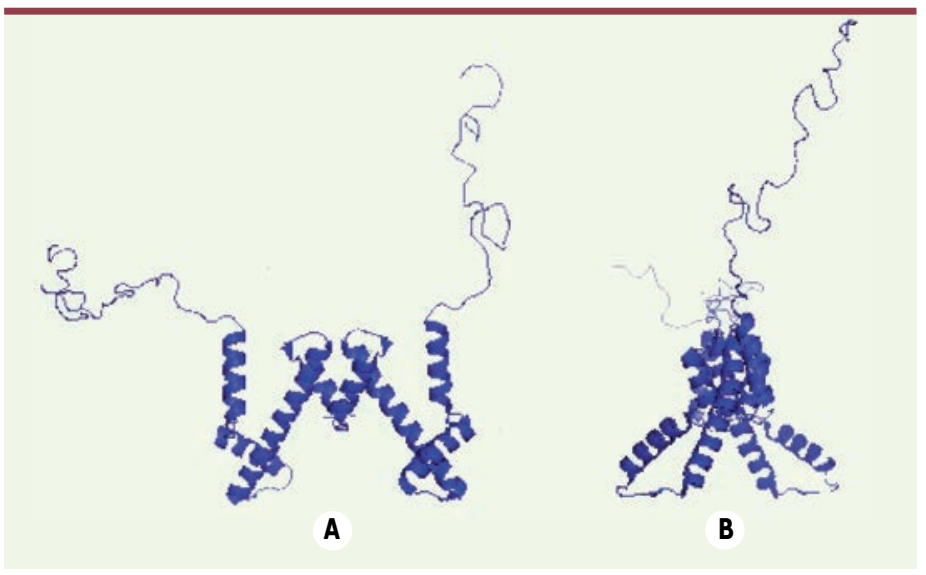

la conformation des NCP. La position de I'ADN nucléosomal était prévue par la structure du cœur protéique [10]. ADN et histones interagissent en 14 positions, toutes les dix paires de bases environ. Au niveau des douze positions les plus centrales, une chaîne latérale d'arginine interagit avec un petit sillon d'ADN par des liaisons hydrogènes, hydrophobes, électrostatiques et des interactions de Van der Waals.

Plus récemment, l'utilisation d'un ADN de 147 pb a permis d'augmenter la résolution à $1,9 \AA$ ([11], fichier pdb $1 \mathrm{KX} 5)$. Cette paire de bases supplémentaire permet de mieux symétriser la particule, l'axe binaire traversant la paire de base centrale et séparant I'ADN nucléosomal en deux moitiés de 73 pb chacune. Cette structure ne modifie pas la précédente organisation au sein des NCP, mais sa meilleure résolution a permis de localiser plus de 3000 molécules d'eau ainsi que certains ions manganèse et chlorure (Figure $3 B$ ). Les molécules d'eau semblent intervenir pour former des ponts entre ADN et protéines via des liaisons hydrogènes.

Différentes structures cristallographiques de NCP comportant soit des histones d'origines différentes (poulet, levure, humain) soit des variants d'histones, dont les séquences et fonctions sont plus divergentes, ont été établies. Pour les NCP dont on dispose d'une structure, leur structure globale reste similaire [12-15]. Le mode de fonctionnement global des NCP est donc certainement universel. Les sites d'interaction histoneshistones sont éventuellement modifiés. Par exemple, dans la structure des NCP de levure [13], un site d'interaction entre les deux dimères $\mathrm{H} 2 \mathrm{~A}-\mathrm{H} 2 \mathrm{~B}$ est absent. De très faibles modifications structurales peuvent néanmoins impliquer des modifications fonctionnelles relativement importantes. La structure des NCP contenant le variant d'histone $\mathrm{H} 2 \mathrm{~A}-\mathrm{Z}$ est par exemple très peu différente de la structure canonique des NCP. La présence de ce variant semble pourtant jouer un rôle central dans la modulation de l'expression des gènes [16].
Figure 2. Repliement de l'histone $\mathrm{H3}$ au sein de la particule cour de nucléosome en vue de face ( $A$ ) et vue de côté (B). Les extrémités amino- et carboxy-terminales sont étendues pour permettre le passage au travers de l'hélice d'ADN.

\section{Les extrémités amino-}

et carboxy-terminales des histones

La plupart des structures cristallographiques de particules cœurs de nucléosome ne positionnent pas précisément les acides aminés des extrémités amino- et carboxy-terminales des histones. En effet, ces extensions ou «queues» ne sont pas structurées. Elles sont mobiles et libres de s'étendre au-delà du cœur des particules (Figure 2). Elles représentent $28 \%$ de la masse totale des histones et contiennent environ un tiers des charges positives de la particule grâce à la présence de nombreux acides aminés basiques (lysine et arginine). Dans la structure établie par Luger et al. en 1997 [9] (Figure 3A), seulement $30 \%$ des acides aminés des « queues » sont positionnés. Dans la structure à $1,9 \AA$ de résolution [11] (Figure 3B), les acides aminés des «queues» sont tous positionnés. Néanmoins, pour les acides aminés les plus terminaux, les facteurs de température («facteurs $B »$ ), indicateurs de désordre n’y sont pas précisés et sont donc certainement élevés.

D’un point de vue structural, certaines de ces extrémités interagissent avec I'ADN qui les entoure et maintiennent ainsi l'intégrité structurale des NCP. Néanmoins, ces extrémités interagissent également avec les particules voisines participant ainsi à l'organisation supramoléculaire de la chromatine. D'un point de vue fonctionnel, ces extrémités font l'objet de modifications covalentes aussi appelées modifications post-traductionnelles. Ces modifications affectent particulièrement les extrémités amino-terminales des histones $\mathrm{H} 3$ et $\mathrm{H} 4$, et leur association à des processus biologiques spécifiques a conduit à proposer l'hypothèse d'un « code histone» [17]. Ce code établirait un lien direct entre la modification de certains résidus des queues des histones et l'état transcriptionnel de la chromatine [18]. Les modifications post-traductionnelles les plus étudiées sont l'acétylation de lysines, la méthylation de lysines et d'arginines, la phosphorylation de sérines et thréonines et l'ubiquitinylation de lysines.

Brièvement, l'acétylation des lysines résulte de la formation d'une liaison covalente entre un groupement acétate et une lysine conduisant ainsi à neutraliser la charge positive des lysines. Cinq lysines appartenant aux queues $\mathrm{H} 3$ et quatre lysines situées sur les queues de H4 sont susceptibles d'être acétylées. In vivo, l'acétylation induite par des acétyl transférase (HAT) diminue les interactions entre NCP voisins provoquant ainsi la décompaction de la chromatine [19]. L'acétylation est réversible. Les lysines acétylées peuvent être désacétylées par des désacétylases. À l'opposé, la méthylation des groupements basiques, lysines et arginines, par des méthyltransférases conserve la charge positive du groupement amine. La réversibilité du processus de méthylation a été mise en évidence récemment. Une enzyme capable de déméthyler des arginines a été découverte [20]. De même, la découverte, in vitro, de l'activité des déméthylases des lysines, nécessitant l'action conjointe de méthylases et déméthylases, laisse 
penser que ce mécanisme existe in vivo [21]. Nous ne détaillerons pas dans cet article la phosphorylation de sites spécifiques de la portion amino-terminale de $\mathrm{H} 3$ qui ajoute une charge négative au groupement phosphorylé, ni l'ubiquitinylation, qui correspond à la liaison d'une protéine de 76 acides aminés sur un acide aminé de H2A ou H2B.

La complexité des mécanismes qui mènent à ces modifications et qui induisent des fonctions particulières au sein de la chromatine dépasse le cadre de cet article et ne peut être explicitée, ici, en détails. De plus, ces différentes modifications ne sont souvent pas activées indépendamment les unes des autres et leur mode de fonctionnement peut être synergique. Par exemple, l'acétylation de la lysine 16 de $\mathrm{H} 4$ en concomitance avec la phosphorylation de la sérine 10 de $\mathrm{H} 3$ amplifie l'activité transcriptionnelle du chromosome X masculin [17]. De même, $\mathrm{H} 3$ et $\mathrm{H} 4$ sont des cibles privilégiées pour la méthylation lorsqu'elles sont acétylées [22]. Enfin, des modifications post-traductionnelles de même nature peuvent entraîner des effets opposés.

\section{La chromatine eucaryote : une organisation dynamique}

Si l'on considère la structure présentée précédemment comme figée, l'enroulement de I'ADN autour de l'octamère d'histone dans les nucléosomes ainsi que la compaction très importante de l'ADN au sein du noyau répriment l'activité transcriptionnelle de la chromatine, I'ADN n'étant pas accessible à la machinerie transcriptionnelle. Pour ces raisons, la chromatine condensée appelée hétérochromatine est généralement considérée comme transcriptionnellement inactive tandis que la chromatine décondensée, l'euchromatine, est, elle, active. Un mode d'organisation dynamique de la chromatine est assuré par plusieurs facteurs [23] : la « respiration » spontanée de l'ADN, le glissement de l'ADN autour des histones par des facteurs de remodelage, les modifications post-traductionnelles des queues des histones, l'incorporation de variants d'histones dans les NCP. Ces vecteurs de plasticité ne sont pas indépendants les uns des autres. Leur activation est liée et peut éventuellement s'avérer synergique.

\section{Ouverture spontanée de l'ADN nucléosomal :}

\section{« respiration 》 de l'ADN}

Des études récentes montrent que la structure des NCP n'est pas figée et qu'il est possible d'observer, notamment in vitro, des mouvements rapides d'ouverture et de fermeture de L'ADN nucléosomal par FRET (fluorescence

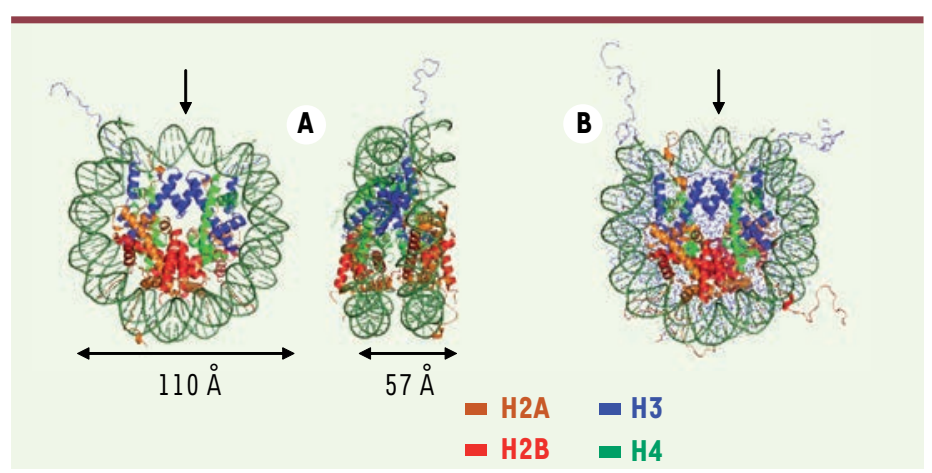

resonance energy transfer) ou FCS (fluorescence correlation spectroscopy) [20-22]. Les nucléosomes restent bien enroulés pendant $250 \mathrm{~ms}$ puis l'ADN s'ouvre pendant des intervalles de temps variant de 10 à $50 \mathrm{~ms}$, assez longs pour rendre l'ADN accessible à des protéines et à des enzymes [24]. Les NCP où l'ADN est bien enroulé sont en équilibre avec des particules dans un état transitoire où l'ADN se déroule pour qu'une protéine puisse éventuellement se fixer à une portion spécifique d'ADN libre.

\section{Remodelage de la chromatine}

Le remodelage de la chromatine est assuré par des complexes protéiques constitués de deux à 12 sousunités [25]. Ces complexes possèdent tous une sous unité ATPase qui leur permet de modifier la structure des NCP en partie grâce à l'énergie libérée lors de l'hydrolyse de l'ATP. On les regroupe par familles. Ils jouent un rôle au niveau de la transcription, de la réplication et de la réparation de l'ADN.

La première famille de facteurs de remodelage est la famille SWI/SNF. Ces complexes comportent 8-15 sous unités dont sept constituent une sous-unité ATPase. Ces complexes augmentent l'accessibilité de l'ADN aux nucléases. Leur action peut être redondante avec celle de l'acétylation des histones. Ils peuvent permettrent l'échange de dimères d'histones H2A/H2B entre NCP. Un autre sous groupe est celui dont les facteurs de remodelage contiennent I'ATPase ISWI. Les complexes ISWI ne comportent que de deux à quatre sous-unités. Le rôle d'ISWI semble être de faire «glisser » les histones sur I'ADN plutôt que d'être impliqué dans la transcription. Les facteurs de remodelage ISWI fonctionnent en dimères. Une troisième famille est celle des complexes NURD dont l'unité ATPase est Mi-2. NURD est impliqué à la fois dans le remodelage et la désacétylation des histones. Mi-2 utilisé seul, in vitro, perturbe les interactions ADNhistones et induit le glissement des NCP.

Différentes hypothèses pour les mécanismes de remodelage des NCP ont été proposées. Pour l'instant, ces mécanismes ne sont pas complètement élucidés. Ces facteurs

Figure 3. Structure cristallographique de la particule cœur de nucléosome. A. Structure cristallographique contenant 146 paires de bases d'ADN, à $2,8 \AA$ de résolution en vue apicale, le long de l'axe de la super-hélice d'ADN et de côté (d'après [7]). B. L'utilisation de 147 paires de bases d'ADN assure la symétrie de la particule permettant ainsi la détermination d'une structure à $1,9 \AA$ de résolution (d'après [9]. Les extrémités amino- et carboxy-terminales des histones, non structurées, sont toutes localisées dans cette dernière structure, mais sont très mobiles. Le pseudo-axe de symétrie binaire, ou axe dyade, est indiqué par une flèche sur les deux structures. Les histones $\mathrm{H} 2 \mathrm{~A}$ sont colorées en orange, $\mathrm{H} 2 \mathrm{~B}$ en rouge, $\mathrm{H} 3$ en bleu et $\mathrm{H} 4$ en vert. 
de remodelage viendraient désolidariser une extrémité d'ADN liée aux histones, provoquant l'obtention de différents types d'états intermédiaires des NCP qui pourraient être instables. Un premier modèle propose que les facteurs de remodelage se lieraient à I'ADN nucléosomal pour induire la formation d'une boucle d'ADN de plusieurs dizaines de paires ou d'une dizaine de paires de base qui se détacheraient du cœur protéique. Cette boucle se propagerait ensuite pour translater l'ADN par rapport aux histones [26]. Un second modèle propose que sous I'action de facteurs de remodelage SWI/SNF, les dimères H2A/H2B ou l'ensemble des histones seraient expulsés du complexe ADN-protéine, pour faciliter le remodelage des NCP [27]. Les facteurs de remodelage semblent également s'accompagner de modifications dans la topologie de l'ADN [28].

L'action des facteurs de remodelage est spécifique. Ils reconnaissent des promoteurs, des séquences de protéines spécifiques, de l'ADN méthylé. II pourrait y avoir une synergie entre les facteurs de remodelage et l'acétylation des histones. Plus particulièrement, la queue d'histone $\mathrm{H} 4$ est nécessaire pour induire l'activité ATPase de NURF et de ISWI [29,30].

\section{Conclusion}

Chez les organismes eucaryotes, la compaction de l'ADN est remarquablement conservée et elle est assurée par son enroulement autour d'histones pour former la particule cœur de nucléosome. Non seulement ce mode d'organisation est capable de confiner l'information génétique en des domaines de très petite taille, mais cette organisation n'est pas figée et reste dynamique. Ainsi, l'ADN est accessible et fonctionnel.

Bien que la particule cœur de nucléosome ait été décrite précisément et sans ambigüité, les niveaux d'organisation supérieurs de l'ADN n'ont pas été explicités et restent très controversés. L'organisation supramoléculaire de l'ADN des nucléosomes jusqu'aux chromosomes reste à déterminer. $\diamond$

\section{SUMMARY}

\section{Structure and dynamic}

of the nucleosome core particle

In eukaryotic cell, a few meters of DNA are compacted in nuclear compartment of a few microns. This high level of compaction is an important way to regulate gene expression. In the present paper, we present a description of the organization of DNA into its first level of compaction: the nucleosome core particle. The structure of the nucleosome has been described at an atomic resolution more than 10 years ago, where DNA is wrapped around an octamer of histones. Post-translational modifications affecting histone tails have been shown to regulate the chromatin degree of compaction and thus the gene expression and regulation. The structure of the NCP is far from being frozen and is highly dynamic. Remodeling factors can induce DNA sliding around the histones, DNA transaction processes such as transcription and replication. $\diamond$

\section{RÉFÉRENCES}

1. Lomberk G, Wallrath L, Urrutia R. The heterochromatin protein 1 family. Genome Biol 2006 ; $7: 228$.

2. Peterson C. The SMC family: novel motor proteins for chromosome condensation? Cell 1994 ; $79: 389-92$.

3. DeLange RJ, Fambrough DM, Smith EL, Bonner J. Calf and Pea Histone IV. II. The complete amino acid sequence of calf thymus histone IV. Presence of egr-N-acetyllysine. J Biol Chem $1969 ; 244: 319-34$.

4. Paedon JF, Wilkins MHF. A super-coil model for nucleohistone. J Mol Biol 1972; 68 : 115-24.

5. Hewish DR, Burgoyne LA. Chromatin sub-structure. The digestion of chromatin DNA at regularly spaced sites by a nuclear deoxyribonuclease. Bioch Bioph Res Com 1973; $52: 504-10$.

6. Kornberg R, Thoma J. Chromatin structure: oligomers of the histones. Science 1974 ; $184: 865-8$.

7. Olins A, Olins D. Spheroid chromatin units ( $v$ bodies). Science $1974 ; 183: 330-2$.

8. Richmond TJ, Finch JT, Rushton B, et al. Structure of the nucleosome core particle at $7 \AA$ resolution. Nature $1984 ; 311: 532-7$.

9. Luger K, Mader AW, Richmond RK, et al. Crystal structure of the nucleosome core particle at $2.8 \AA$ resolution. Nature $1997 ; 389: 251-60$.

10. Arents G, Burlingame RW, Wang B, et al. The nucleosomal core histone octamer at $3.1 \AA$ resolution: a tripartite protein assembly and a left-handed superhelix. Proc Natl Acad Sci USA $1991 ; 88: 10148-52$.

11. Davey CA, Sargent DF, Luger K, Maeder AW, Richmond TJ. Solvent mediated interactions in the

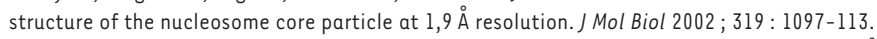

12. Harp JM, Hanson BL, Timm DE, Bunick GJ. Asymmetries in the nucleosome core particle at $2.5 \AA$ resolution. Acta Cryst Section D $2000 ; 56: 1513-34$.

13. White $C$, Suto R, Luger K. Structure of the yeast nucleosome core particle reveals fundamental changes in internucleosome interactions. EMBO J 2001; $20: 5207-18$.

14. Suto RK, Clarkson MJ, Tremethick DJ, Luger K. Crystal structure of a nucleosome core particle containing the variant histone H2A.Z. Nat Struct Mol Biol $2000 ; 7$ : 1121-4.

15. Tsunaka Y, Kajimura N, Tate S, Morikawa K. Alteration of the nucleosomal DNA path in the crystal structure of a human nucleosome core particle. Nucleic Acids Res 2005 ; 33 : 3424-34.

16. Guillemette B, Gaudreau L. H2A.Z : un variant d'histone qui orne les promoteurs des gènes. Med Sci (Paris) 2006; $22: 941-6$.

17. Strahl BD, Allis CD. The language of covalent histone modifications. Nature $2000 ; 403: 41-5$. 18. Lacoste N, Côté J. Le code épigénétique des histones. Med Sci (Paris) 2003 ; 19 : 955-9.

19. Miotto B, Struhl K. De la régulation du génome à la progression tumorale : acétylation de la lysine 16 de l'histone H4. Med Sci (Paris) 2007 ; 23 : 735-40.

20. Cheung P, Lau P. Epigenetic regulation by histone methylation and histone variants. Mol Endocrinol $2005 ; 19: 563-73$.

21. Lan F, Nottke AC, Shi Y. Mechanisms involved in the regulation of histone lysine demethylases. Curr Op Cell Biol $2008 ; 20: 316-25$.

22. Annunziato AT, Eason MB, Perry CA. Relationship between methylation and acetylation of arginine-rich histones in cycling and arrested HeLa cells. Biochemistry $1995 ; 34: 2916-24$.

23. Luger K. Dynamic nucleosomes. Chrom Res $2006 ; 14: 5-16$.

24. Li G, Levitus M, Bustamante C, Widom J. Rapid spontaneous accessibility of nucleosomal DNA. Nat Struct Mol Biol $2004 ; 12: 46-53$.

25. Becker PB, Horz W. ATP-dependent nucleosome remodeling. Ann Rev Biochem 2002 ; $71: 247-73$.

26. Langst G, Becker PB. ISWI induces nucleosome sliding on nicked DNA. Mol Cell 2001; $8: 1085-92$.

27. Peterson C, Tamkun J. The SWI/SNF complex: a chromatin remodeling machine? Trends Biol Sci $1995 ; 20: 143-6$.

28. Gavin I, Horn P, Peterson C. SWI/SNF chromatin remodeling requires changes in DNA topology. Mol Cell 2001; $7: 97-104$.

29. Clapier C, Längst G, Corona D, et al. Critical role for the histone $\mathrm{H} 4 \mathrm{~N}$ terminus in nucleosome remodeling by ISWI. Mol Cell Biol $2001 ; 21: 875-83$.

30. Hamiche A, Kang JG, Dennis C, et al. Histone tails modulate nucleosome mobility and regulate ATP-dependent nucleosome sliding by NURF. Proc Natl Acad Sci USA 2001 ; 98 : 14316-21.

\section{TIRÉS À PART}

S. Mangenot 